\title{
Precision Analysis of Color Measurement by Different Spectrophotometers
}

\author{
Hongying Yang ${ }^{1,2} a^{*}$, Shiyin Ding ${ }^{1, b}$, Pengfei Jiang ${ }^{1, c}$ \\ Zhihui Yang ${ }^{1, d}$, Ge Zhang ${ }^{1,3, e}$, and Shizhong Cui ${ }^{1,2, f}$ \\ ${ }^{1}$ Zhongyuan University of Technology, Zhengzhou, 450007, China \\ ${ }^{2}$ Collaborative Innovation Center of Textile and Garment Industry, Henan province, China \\ ${ }^{3}$ Jiangnan University, Wuxi, 214122, China \\ awebyhy@163.com, bding_shiyin@foxmail.com, 'pfjiang@hotmail.com \\ `wynnlove@hotmail.com, èzhangge08105@163.com, ’szcui@zzti.edu.cn
}

\begin{abstract}
Keywords: Color measurement, spectrophotometer, precision analysis, color difference, reflectance, range, standard deviation

Abstract. Photometric accuracy and precision are the most important technical parameters of spectrophotometers. In this paper, our three instruments, that is Datacolor SF600, X-Rite Color i7 and Varian Cary 5000 UV-VIS-NIR spectrophotometer, were inspected on their photometric precision for color measuring. Five precision test methods basing on color difference or reflectance were utilized. Experiments and computations show coincident results, which are helpful for further analysis of their applicability and for corresponding utilization in future study.
\end{abstract}

\section{Introduction}

There are three high precision desktop spectrophotometers available in our laboratory which can be conveniently utilized to measure the reflectance spectrum, the "fingerprint" of the color of opaque object. They are Datacolor SF600, X-Rite Color i7 and Varian Cary 5000 UV-VIS-NIR spectrophotometers, which are all well-known optical instruments in the world. Our three instruments have great difference. The Datacolor SF600 is old, imported in 2004 and has some pollution in its integrating sphere; while X-Rite Color i7 and Varian Cary 5000 are new, installed in 2014. Besides, the three instruments have different optical path and geometry so they yield different color measuring results [1]. Photometric accuracy and precision, wavelength accuracy and precision are the most important technical parameters of the spectrophotometer. Accuracy is determined by the systematic error, while the precision is determined by the random error [2]. This paper focuses on inspecting the photometric precision of our three instruments in order to find out their applicability for further study of color related projects.

Precision is the consistent degree of the results in repeating measurement [3-5]. Precision has two classifications - repeatability and reproducibility. Repeatability is an evaluation of variations in the measured values with a given instrument and sampled over a specific period. Reproducibility is an evaluation of variations in the measured values with changing conditions such as the operator, the laboratory, the temperature, or any other condition that might be subject to change [6]. Aiming to compare the precisions of our three instruments, we focus on the repeatability of the precision.

Each instrument manufacturer has their respective precision test method. For color measurement, color difference and reflectance are often utilized for precision evaluation [7]. Datacolor adopts both color difference with setting standard sample and range of reflectance; while X-Rite applies root mean square of color difference. Besides, the standard deviation is usually calculated to assess the deviation of repeated measurement [8]. In order to acquire the maximum color difference caused by random error of the instruments, maximum color difference is also computed to estimate the photometric precision in this study. 


\section{Experimental}

Every instrument was switched on for 30 minutes before calibrating and measuring in order to reach a steady state. The measurement conditions were set as same or alike as possible except the intrinsic differences among the instruments. $\mathrm{D}_{65}$ illuminant, 10 degree visual field, specular reflection and ultra-violet included were set as the same in all tests [8]. The illuminant/observer conditions of Datacolor and X-Rite are $\mathrm{d} / 8^{\circ}$, while Cary 5000 is $3^{\circ} 20^{\prime} / \mathrm{d}$. The apertures applied were $9 \mathrm{~mm}, 25 \mathrm{~mm}$ and $16 \mathrm{~mm}$ for Datacolor, X-Rite and Cary respectively according to the practical situation.

Both Datacolor and X-Rite used white tile in their precision test, so in this experiment a white tile from X-Rite instrument were employed. According to Datacolor and X-Rite's precision test method, the white tile was measured 20 times on a fixed position (without moving the instrument and the tile) by the three instruments separately. The time intervals between two adjacent measurements were about 10 seconds.

\section{Precision Calculation and Analysis}

Measured spectral reflectance of the white tile by the three instruments were exported and then imported into MATLAB, which were employed to performance all the precision calculations.

Method 1: Color Difference with Setting Standard Sample. In this method, the first measuring result was set as that of the standard sample. Color differences between the standard sample and all the other 19 measurements were calculated. The basic color difference $\mathrm{DE}_{\mathrm{CIELAB}}[5,6,8,9]$ was computed and the results were plotted in Fig. 1.

From Fig. 1, compare the largest $\mathrm{DE}_{\mathrm{CIELAB}}$, Datacolor $(0.1407)>$ Cary $5000(0.0700)>$ X-Rite (0.0431). The DE $\mathrm{E}_{\text {CIELAB }}$ of Datacolor is increasing with times, while the DE $\mathrm{CIELAB}_{\mathrm{B}}$ of the Cary5000 and $\mathrm{X}$-Rite are normally fluctuating. Further compare the average $\mathrm{DE}_{\mathrm{CIELAB}}$, also Datacolor $(0.0570)>$ Cary 5000 (0.0406) $>$ X-Rite (0.0281). Hence, from this method, the photometric precisions of our three instruments are X-Rite $>$ Cary5000 $>$ Datacolor. This precision test method can clearly show the stability of the instrument over time.

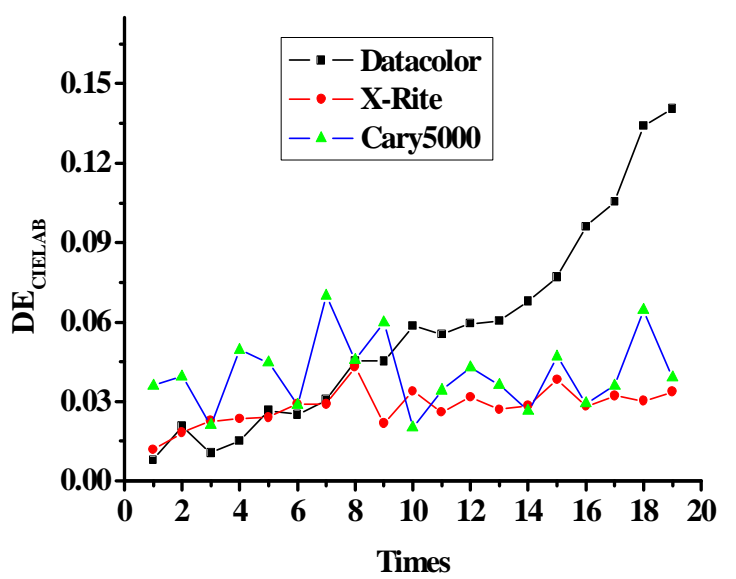

Table 1 Maximum color difference

\begin{tabular}{ccc}
\hline Instrument & $\begin{array}{c}\text { Max } \\
\mathrm{DE}_{\text {CIELAB }}\end{array}$ & $\begin{array}{c}\mathrm{Max} \\
\mathrm{DE}_{\mathrm{CMC}(1: 1)}\end{array}$ \\
\hline $\begin{array}{c}\text { Datacolor } \\
\text { SF600 }\end{array}$ & 0.1430 & 0.1075 \\
$\begin{array}{c}\text { Varian } \\
\text { Cray 5000 }\end{array}$ & 0.0700 & 0.0906 \\
$\begin{array}{c}\text { X-Rite } \\
\text { Color i7 }\end{array}$ & 0.0431 & 0.0574 \\
\hline
\end{tabular}

Fig. 1 Color difference with set standard sample

Method 2: Root Mean Square of Color Difference. This method is to calculate the color differences between two adjacent measurements, and then the root mean square values of all the color differences is calculated. This method can effectively study the repeatability of the instrument in very short-term measurements.

For each of the three instruments, $19 \mathrm{DE}_{\mathrm{CIELAB}}$ values were computed. The root mean square values of their DE $\mathrm{CIELAB}_{\mathrm{B}}$ are Datacolor (0.0149) < X-Rite (0.0150) < Cary5000 (0.0264). It's obvious that our Datacolor and X-Rite have similar stability in adjacent measurement, slightly better than our Cary5000. The results agree well with their fluctuations as shown in Fig 1. 
Method 3: Maximum Color Difference. Calculate the color differences between a measurement and all the other measurements and choose the maximum. This method is sensitive to the measurement with a large error. In this test, there are totally $190 \mathrm{DE}_{\mathrm{CIELAB}}$ and $380 \mathrm{DE}_{\mathrm{CMC}(1: 1)}$ values. The maximum $\mathrm{DE}_{\mathrm{CIELAB}}$ and $\mathrm{DE}_{\mathrm{CMC}(1: 1)}$ are listed in Table 1 .

From Table 1, it's obvious that the order of the maximum $\mathrm{DE}_{\mathrm{CIELAB}}$ and $\mathrm{DE}_{\mathrm{CMC}(1: 1)}$ are Datacolor $>$ Cary $5000>$ X-Rite. These maximum color difference are larger than that in Color Difference with Setting Standard Sample, because in this method every sample have a chance to be the standard sample and there are totally $190 \mathrm{DE}_{\mathrm{CIELAB}}$ and $380 \mathrm{DE}_{\mathrm{CMC}(1: 1)}$ values instead of $19 \mathrm{DE}_{\mathrm{CIELAB}}$ values.

Method 4: Range of Reflectance. This method is to calculate the range of reflectance, that is, the difference between the maximum and the minimum of the reflectances among the 20 measurements. According to Datacolor's method, the ranges of the reflectance in the band of $400 \mathrm{~nm}$ to $700 \mathrm{~nm}$ are used to evaluate the instruments' precision. The ranges of reflectance from our three instruments were calculated and displayed in Fig. 2.

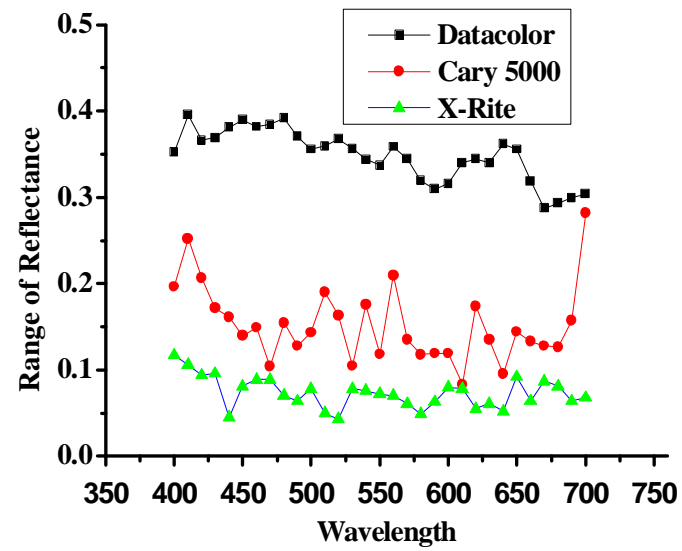

Fig. 2 Range of reflectance

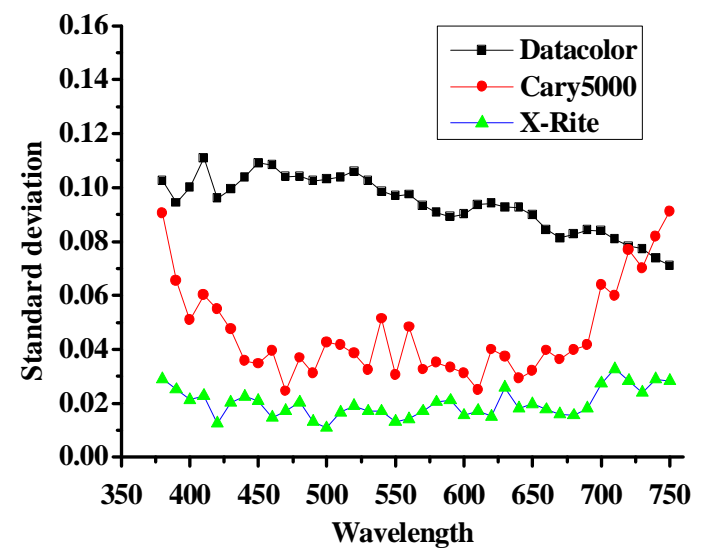

Fig. 3 Standard deviation of Reflectance

From Fig. 2, it is clear that the reflectance ranges of the three instruments at each wavelength are Datacolor $>$ Cary5000 $>$ X-Rite. The average reflectance ranges are Datacolor $(0.348)>$ Cary5000 $(0.152)>$ X-Rite $(0.073)$. This method can reflect the biggest change in reflectance, but each wavelength reflectance range is not obtained in the same test. Therefore, the results can reflect the precision of the instrument as a whole, rather than the precision of each measurement.

Method 5: Standard Deviation of Reflectance. The standard deviation reflects the discrete situation of testing, so the deviation of reflectance can be represented by its standard deviation. The standard deviations of the reflectance at each wavelength from the three instruments were computed and exhibited in Fig. 3.

From Fig. 3, it can be seen that among our three instruments, X-Rite has the lowest reflectance deviation, X-Rite the second lowest, followed by Datacolor. The average values of reflectance standard deviation are Datacolor (0.0939) > Cary5000 (0.0462) > X-Rite (0.01988).

\section{Discussion}

For our three spectrophotometers, the five photometric precision examination methods gave the same results, that is, X-Rite Color i7 > Varian Cary $5000>$ Datacolor SF600. Repeatability should be on the order of 0.1 CIELAB color difference [6]. The maximum DE CIELAB $_{\text {are } 0.04,0.07}$ and 0.14 for X-Rite, Cary 5000 and Datacolor, so they all have acceptable precision.

Both Datacolor SF600 and X-Rite Color i7 are special color measuring instrument, while Cary 5000 is a ordinary spectrophotometer which can be used for color measurement with its integrating sphere attachment. The Datacolor SF600 has the lowest precision among these instruments, which is in the expectation because it has been used for about ten years and polluted to some extent. However, our X-Rite Color i7 has better precision than our Cary 5000 which made us think about. Cary 5000 has 
strong functions and high precision and has the same reputation as X-Rite does. However, X-Rite Color 17 is designed for color measurement while the Cary 5000 doesn't which can be utilized for color measurement with its attachment. Datacolor and X-Rite can be used only in the band of visual light and give data at $10 \mathrm{~nm}$ intervals, while Cary 5000 can be used in the range of ultraviolet-visible-near infrared and output data at $1 \mathrm{~nm}$ intervals. The light source of Datacolor and X-Rite are pulsed-xenon lamp with high energy in the visible region, while Cary 5000 uses a slightly lower energy tungsten lamp in the visible region. Datacolor's and X-Rite's integrating spheres are $152 \mathrm{~mm}$ in diameter while Cary 5000 's is $110 \mathrm{~mm}$. Besides, there are many other differences among the three instruments which also influence their precisions such as the coating material and the open area of the integrating sphere, the sensitivity of the receiver, the monochromator, the geometry and so on. Anyway, these precision examination experiments show clear and consistent results and help us in further analysis of their applicability and corresponding utilization in our future study.

\section{Conclusions}

In order to evaluate the precision of our three different instruments on color measuring, Datacolor SF600, X-Rite Color i7 and Varian Cary 5000, five methods are adopted and the computing and analysis results show the same results that X-Rite give the best precision, Cary 5000 have very good precision too, followed by Datacolor. Explanations were given according to their present situation and inherent difference.

Five precision examination methods have their respective features; among them, the method of Standard Deviation of Reflectance is more direct, simple and general, while the methods with color difference can give a color difference scale which is more visual for color measurement. All the five methods yield coincident results in this study.

\section{Acknowledgements}

This work is financially supported by National Natural Science Foundation (61440064), and Scientific Research Foundation for ROCS, SEM and International Cultivation of Henan Advanced Talents.

\section{References}

[1] AATCC EP6-2008, Instrumental color measurement, 2008.

[2] B. Sun, Principal technical indexes of spectrophotometer and their testing methods, J. Analytical Instrumentation, 1 (2007) 53-56. (In Chinese)

[3] W.H. Lian, Precision of spectrophotometer and their deviation, J. Printing Quality and Standardization, 2 (2008) 37-40. (In Chinese)

[4] ASTM-E2214-2013, Standard practice for specifying and verifying the performance of color-measuring instruments (2013).

[5] DIN 55600-2008, Testing of pigments-determination of the significance of color differences of surface color according to the CIELAB-formula-examples for calculation and test report (2008).

[6] D. Malacara, Color Vision and Colorimetry: Theory and Applications, second ed., SPIE, Bellingham, 2011

[7] JJF 1001-1998, General measurement terms and definitions (1998). (In Chinese)

[8] W.J. Hu, S.Q. Tang, Z.F. Zhu, The principle and application of modern color technology, Beijing Institute of Technology Press, Beijing, 2007. (In Chinese)

[9] Z.L. Dong, B.H. Zheng, G.F. Hu, J.Y. Liu, Colorimetry and computer color matching, second ed., China Textile and Apparel Press, Beijing, 2007. (In Chinese) 